\title{
High-throughput prediction of eucalypt lignin syringyl/guaiacyl content using multivariate analysis: a comparison between mid-infrared, near-infrared, and Raman spectroscopies for model development
}

\author{
Jason S Lupoi ${ }^{1,2^{*}}$, Seema Singh ${ }^{2,3}$, Mark Davis ${ }^{4,5}$, David J Lee ${ }^{6}$, Merv Shepherd ${ }^{7}$, Blake A Simmons ${ }^{1,2,3}$
}

and Robert J Henry ${ }^{1}$

\begin{abstract}
Background: In order to rapidly and efficiently screen potential biofuel feedstock candidates for quintessential traits, robust high-throughput analytical techniques must be developed and honed. The traditional methods of measuring lignin syringyl/guaiacyl $(\mathrm{S} / \mathrm{G})$ ratio can be laborious, involve hazardous reagents, and/or be destructive. Vibrational spectroscopy can furnish high-throughput instrumentation without the limitations of the traditional techniques. Spectral data from mid-infrared, near-infrared, and Raman spectroscopies was combined with S/G ratios, obtained using pyrolysis molecular beam mass spectrometry, from 245 different eucalypt and Acacia trees across 17 species. Iterations of spectral processing allowed the assembly of robust predictive models using partial least squares (PLS).

Results: The PLS models were rigorously evaluated using three different randomly generated calibration and validation sets for each spectral processing approach. Root mean standard errors of prediction for validation sets were lowest for models comprised of Raman (0.13 to 0.16 ) and mid-infrared (0.13 to 0.15) spectral data, while near-infrared spectroscopy led to more erroneous predictions (0.18 to 0.21$)$. Correlation coefficients ( $r$ ) for the validation sets followed a similar pattern: Raman (0.89 to 0.91), mid-infrared (0.87 to 0.91), and near-infrared (0.79 to 0.82). These statistics signify that Raman and mid-infrared spectroscopy led to the most accurate predictions of $S / G$ ratio in a diverse consortium of feedstocks.

Conclusion: Eucalypts present an attractive option for biofuel and biochemical production. Given the assortment of over 900 different species of Eucalyptus and Corymbia, in addition to various species of Acacia, it is necessary to isolate those possessing ideal biofuel traits. This research has demonstrated the validity of vibrational spectroscopy to efficiently partition different potential biofuel feedstocks according to lignin S/G ratio, significantly reducing experiment and analysis time and expense while providing non-destructive, accurate, global, predictive models encompassing a diverse array of feedstocks.
\end{abstract}

Keywords: Biomass, Raman spectroscopy, Near-infrared spectroscopy, Fourier-transform infrared spectroscopy, High-throughput, Multivariate analysis, Lignin S/G

\footnotetext{
* Correspondence: jslupoi@|bl.gov

${ }^{1}$ Queensland Alliance for Agriculture and Food Innovation, University of

Queensland, 306 Carmody Road, St. Lucia, QLD 4072, Australia

${ }^{2}$ Joint BioEnergy Institute, Lawrence Berkeley National Laboratory, 5885 Hollis

Street, Emeryville, CA 94608, USA

Full list of author information is available at the end of the article
} 


\section{Background}

Second-generation biofuels from lignocellulosic biomass have been progressively explored as plausible pathways to relinquishing global dependency on greenhouse gasemitting fossil fuels [1-3]. Given the multitude of possible plants, the development of high-throughput analytical techniques capable of screening large arrays of feedstocks is paramount to isolating ideal candidates for biofuel and biochemical research and production. The measurement of biomass phenotypic parameters such as chemical composition, enzymatic hydrolysis sugar release, and the ratio of syringyl (S)-to-guaiacyl (G) lignin moieties can aid in identifying biomass species possessing traits found to play important roles in diminishing biomass recalcitrance. The effects different feedstock traits have on cell wall deconstruction are multifaceted, and no one superlative characteristic has been identified. Therefore, the collective highthroughput measurements of various traits can rapidly illuminate plants of interest. For example, Sykes et al. used high-throughput pyrolysis molecular beam mass spectrometry (pyMBMS) to screen approximately 800 poplar trees based on lignin content and S/G ratio [4].

Lignin is a three-dimensional, structurally complex, biopolymer comprised of phenylpropanoid units, designated as $\mathrm{S}, \mathrm{G}$, and p-coumaryl $(\mathrm{H})$ components $[5,6]$. The ratio of S- to G-lignin provides a pivotal parameter for gauging the expected chemical reactivity of delignifying plant cell walls and for determining the energy requirements for pulping and bleaching feedstocks [7-9]. The correlation of S/G ratios to monomeric sugar release following hydrolysis of biomass has been explored, revealing conflicting results $[7,10,11]$. A slight decrease in the $S / G$ ratio of hybrid poplar was shown to improve the rate of dilute acid hydrolysis [7]. While counter to the authors' original hypothesis, the results demonstrate the applicability of using S/G ratios as an indicator of monomeric sugar yield. When juxtaposed with other correlative studies of $S / G$ ratio and saccharification yield, high S/G ratios have occasionally resulted in the release of larger quantities of monomeric sugars $[10,11]$. Thus, although the exact effect of lignin S/G ratio on enzymatic hydrolysis of polysaccharides has not been elucidated, this metric has proven to be integral to understanding the role lignin structure plays in deconstructing biomass.

The techniques traditionally employed to determine lignin S/G ratios include wet chemistry methods like nitrobenzene oxidation or thioacidolysis, and instrumental approaches such as nuclear magnetic resonance, gas chromatography/ mass spectrometry (GC/MS), pyrolysis GC/MS (pyGCMS), and pyMBMS [12]. These methods can be expensive, laborintensive, time-consuming, destructive, and/or utilize toxic reagents such as boron trifluoride etherate.

Vibrational spectroscopy, including Raman, near-infrared (NIR), and mid-infrared (MIR) has been shown to provide non-destructive, high-throughput, qualitative and quantitative assessments of lignin S/G ratios (Table 1) [13-26]. Typically, the spectral data are combined with analytical results from a traditional technique (pyrolysis/mass spectrometry, thioacidolysis/gas chromatography) for a subset of the samples, and the use of multivariate analysis, including principal component analysis (PCA) and partial least squares regression (PLS) allows the formation of robust models capable of classifying and predicting the analytes for the remaining samples. While many options have been explored in the search for robust, high-throughput analytical techniques to efficiently screen biomass via multivariate modeling, attempts to compare one lab's results with another can be enervating due to the lack of standardization in model construction and in the statistics reported for model critique (Table 1). In order to accurately showcase the predictive power of these instrumental methods, it is imperative for forthcoming literature to include the statistics resultant from the rigorous validation of multivariate models.

Raman spectroscopy measures the amount of photon scattering from a molecule when irradiated with an excitation source, such as a laser [27]. The peaks in a Raman spectrum are characteristic of specific chemical bond vibrational modes, allowing qualitative structural assessments of the analyte as well as quantitation using peak heights and areas. Sample preparation is undemanding, the methodology does not destroy samples, and analytes in aqueous and complex matrices can routinely be evaluated, making Raman spectroscopy a robust, versatile, analytical tool. The use of Raman spectroscopy for the assembly of multivariate models capable of predicting biofuel traits has not been systematically explored, although recent instrumental advances such as the robust, hand-held, field-portable devices, make Raman spectroscopy an attractive option for further application for biomass analysis [28,29].

Fourier-transform (FT) Raman spectroscopy coupled with thioacidolysis has been used to develop PLS models for predicting lignin S/G ratios [16]. Fifty-five Eucalyptus globulus and E. camaldulensis samples were used to create a calibration matrix, while 25 randomly selected samples comprised the prediction set. The authors report a correlation coefficient ( $r$ ) of 0.935 and a standard error of prediction (SEP) of 0.32 using a five factor model. In another study, assessing five different Eucalyptus species, predictive models of lignin S/G ratio using FT-Raman and PLS revealed slightly less accurate models $(r=0.919$, SEP $=$ 0.35 ), perhaps due to the inclusion of a wider variety of feedstocks [23]. However, due to the insertion of these other species, the authors were able to successfully build a more diverse model, capable of predicting traits from both heart- and sapwood, and differences in tree age.

FT-Raman spectral deconvolution between 1220 and $1530 \mathrm{~cm}^{-1}$ has been employed to determine lignin S/G 
Table 1 Evaluation of literature multivariate models for lignin S/G prediction

\begin{tabular}{|c|c|c|c|c|c|c|c|c|c|c|c|c|}
\hline Method & $\begin{array}{c}\text { \# of } \\
\text { samples }\end{array}$ & $\mathrm{SEC}^{1}$ & $\mathrm{SEP}^{2}$ & RM-SEC ${ }^{3}$ & RM- SECV ${ }^{4}$ & $\begin{array}{l}\text { Random } \\
\text { Val. Set }\end{array}$ & RM-SEP 5 & $\mathrm{r}-\mathrm{Val}{ }^{6}$ & $R^{2} \mathrm{Cal}^{7}$ & $R^{2} \mathrm{Val}^{8}$ & $F^{9}$ & Ref \\
\hline Raman & 10 & - & - & $0.02(\mathrm{~S}) 0.04(\mathrm{G})$ & - & $\mathrm{N}$ & $0.04(\mathrm{~S}) 0.04(\mathrm{G})$ & $0.993(S) 0.992(G)$ & $0.996(\mathrm{~S}) 0.997(\mathrm{G})$ & $0.985(\mathrm{~S}) 0.986(\mathrm{G})$ & - & [15] \\
\hline Raman & 55 (Cal) 25 (Val) & 0.07 & 0.32 & - & - & Y & - & 0.935 & 0.996 & 0.874 & 5 & [16] \\
\hline Raman & 63 (Cal) 30 (Val) & 0.32 & 0.35 & - & - & Y & - & 0.919 & 0.887 & 0.845 & 2 & [23] \\
\hline Raman & 9 & - & - & - & - & $N$ & - & - & - & 0.983 & - & [20] \\
\hline MIR & 5 & - & - & - & - & - & - & - & - & - & - & [14] \\
\hline MIR & 5 & - & - & - & - & - & - & - & - & - & - & [24] \\
\hline MIR & 65 & - & - & - & - & - & - & - & - & $0.91-0.98$ & - & [25] \\
\hline MIR & 15 & - & - & - & - & - & - & - & - & - & - & [26] \\
\hline NIR & 42 (Cal) 36 (Val) & - & - & - & $0.025-0.033$ & Y & $0.025-0.036$ & $0.959-0.980$ & $0.95-0.97$ & $0.92-0.96$ & $3-5$ & [13] \\
\hline NIR & 267 (Cal) & - & - & $\begin{array}{l}0.176(\mathrm{~S}) 0.202 \\
(\mathrm{G}) 0.005(\mathrm{H})\end{array}$ & $\begin{array}{c}0.201(S) 0.202 \\
(G) 0.005(H)\end{array}$ & $N$ & - & $\begin{array}{c}0.979(\mathrm{~S}) 0.979(\mathrm{G}) \\
0.843(\mathrm{H})\end{array}$ & $\begin{array}{l}0.968(S) 0.957 \\
(G) 0.731(H)\end{array}$ & $\begin{array}{c}0.958(\mathrm{~S}) 0.957(\mathrm{G}) \\
0.710(\mathrm{H})\end{array}$ & 8 & [17] \\
\hline NIR & 135 (Cal) 45 (Val) & - & 0.124 & - & 0.121 & Y & - & 0.686 & 0.583 & 0.47 & $5-7$ & [19] \\
\hline NIR & 26 (Cal) 8 (Val) & 0.26 & 0.3 & - & - & Y & - & 0.938 & 0.96 & 0.88 & 6 & [22] \\
\hline
\end{tabular}

\section{${ }^{1}$ Standard Error of Calibration.}

Standard Error of Prediction.

Root Mean Standard Error of Calibration.

Root Mean Standard Error of Cross-Validation.

Root Mean Standard Error of Prediction.

Coefficient of Correlation for Validation Set.

Coefficient of Determination for Calibration Set.

Coefficient of Determination for Validation Set.

\#of Factors.

Italicized values calculated by the authors.

MIR = mid-infrared spectroscopy, NIR = near-infrared spectroscopy, $\mathrm{Cal}=$ calibration set, $\mathrm{Val}=$ validation set, $\mathrm{S}=$ syringyl, $\mathrm{G}=$ guaiacyl, $\mathrm{H}=p$-hydroxyphenol, $\mathrm{Y}=\mathrm{yes}, \mathrm{N}=\mathrm{no}$. 
ratios in nine feedstocks including Arabidopsis thaliana and E. globulus [20]. The authors developed a calibration equation to compare results obtained via pyGCMS with good correlation (coefficient of determination, $R^{2}=$ 0.983), despite observed overlap between the spectral regions designated as $\mathrm{S}, \mathrm{G}$, or $\mathrm{H}$ lignin signatures, and vibrational modes due to polysaccharides cellulose and xylan. Near-infrared, dispersive, multichannel Raman spectroscopy coupled with thioacidolysis/GCMS data allowed the quantitation of S and G lignin content in 10 feedstocks, with emphasis on herbaceous plants [15]. A principal component regression model was generated that accurately predicted the $S / G$ ratio for the majority of plants studied $\left(R^{2}=0.985-0.986\right.$, root mean standard error of prediction $($ RMSEP $)=0.02-0.04)$.

Mid-infrared (MIR) spectroscopy provides complementary information to Raman spectroscopy due to the difference in selection rules. In Raman spectroscopy, a change in the polarizability of an electron cloud is required for a molecule to be 'active', while in MIR spectroscopy, there must be a change in dipole [30]. Thus, the use of both techniques in tandem can present more complete structural assessments of analytes. The use of MIR spectroscopy to analyze biomass has been more frequently employed to obtain qualitative structural information rather than as a quantitative tool for development of multivariate predictive models, in part due to the strong absorption of water, making analysis of aqueous and biological samples difficult. Some recent endeavors employing MIR spectroscopy have explored quantifying lignin S/G ratios. del Rio et al. used MIR spectroscopy to develop a technique for estimating S/G ratios in lignin isolated from hemp, flax, jute, sisal, and abaca feedstocks [14]. Following the selected spectral processing of resolution enhancement, smoothing, and baseline correction, peak intensities at 1327 and $1271 \mathrm{~cm}^{-1}$ were used as a marker for S- and G-lignin, respectively. The calculated S/G ratios were found to agree with pyGCMS.

To date, the most widespread vibrational spectroscopy screening methods employ NIR spectroscopy. The straightforward instrumentation and sample handling in NIR spectroscopy has permitted the screening of large arrays of biomass feedstocks to identify those possessing the key traits necessary for efficient biofuel production including lignin S/G ratios [13,17,19,22]. Unlike Raman or MIR spectroscopies that characterize fundamental vibrational modes, NIR measures overtone and combination bands associated with $\mathrm{C}-\mathrm{H}, \mathrm{N}-\mathrm{H}, \mathrm{O}-\mathrm{H}$, and $\mathrm{S}-\mathrm{H}$ moieties [31]. These spectral features are subtle and require spectral deconvolution or multivariate analysis for the elucidation of useful parameters.

Thioacidolysis/GCMS results were coupled with NIR spectra to develop a PLS model for predicting S/G ratios in transgenic aspen [22]. The authors report a calibration $R^{2}$ of 0.96 and a standard error of calibration (SEC) of 0.26 using six factors and 26 spectra. Eight spectra were used to validate the PLS model, resulting in a $R^{2}$ of 0.88 , and SEP of 0.3. NIR spectra of 267 wild and transgenic poplar samples were also conjoined with $S$, $\mathrm{G}$, and $\mathrm{H}$ lignin fractions measured using a streamlined, higher-throughput thioacidolysis protocol [17]. The resultant PLS model used 8 factors to explain the variance and a full cross-validation to determine the predictive capacity. The authors report $R^{2}$ values 0.958 (S), $0.957(\mathrm{G})$, and $0.710(\mathrm{H})$ for model prediction and cross-validation RMSE values of 0.201 (S), 0.202 (G), and $0.005(\mathrm{H})$. A randomly selected validation set was not employed to more rigorously test the model's validity. Finally, NIR spectra and analytical pyrolysis were used to build PLS models to predict the S/G ratios of E. globulus trees [13]. The authors randomly selected 42 of 78 samples to compose the calibration model, using the remaining 36 samples for validation. A full cross-validation indicated that 3 to 5 factors were sufficient to explain the variance in the data, depending on the type of spectral processing utilized. The prediction $R^{2}$ values ranged from 0.92 to 0.96 while the RMSEP values were between 0.024 and 0.036 .

The nomination of appropriate second-generation biofuel candidates is paramount to reducing consumption of nonrenewable energy sources $[1,32,33]$. Eucalypts possess attractive characteristics that increase their candidacy for further biofuel production studies, including the ability to grow on marginal land, in low nutrient and degraded soil, their proficiency of growing rapidly and in a wide variety of climates, the coppicing of many eucalypt species preventing the need to replant the tree, and the well-known understanding of breeding, growing, processing, and chemical composition of the biomass [34].

This manuscript describes the marriage of multivariate analysis, vibrational spectroscopy, and pyMBMS data to assemble PLS models capable of partitioning diverse eucalypts and Acacias according to lignin S/G ratio. MIR, NIR, and Raman spectroscopic methods are illustrated, including spectral acquisition and processing, and the resultant models are critiqued statistically in order to elect which technique(s) will provide the most accurate prediction of lignin S/G ratios. It is demonstrated that MIR and Raman spectroscopy, although sporadically portrayed in current literature, are capable of providing powerful high-throughput analytical tools for lignin S/G analysis. Additionally, while many previous studies have focused on one type of biomass (E. globulus, or transgenic and wild-type poplar), the global models constructed in this study incorporate three genera encompassing 17 different species of biomass, including wild-type and hybrid Corymbia, which, to the authors' knowledge, have not been extensively characterized. 


\section{Results and discussion Compilation of reference set}

The reference set of wood samples selected for pyMBMS analysis was designed to represent the greatest amount of variance between the diverse collections of plants. This was achieved by performing a PCA of the MIR and NIR spectral data. In PCA, samples are classified according to similarity, thus when considering a scores plot when two samples have neighboring coordinates they are more similar; the farther they are from each other, the more dissimilar. A Hotelling $\mathrm{T}^{2}$ ellipse can be drawn around the samples to illustrate which samples lay farthest from the mean sample. The samples that fell outside or near the periphery of the ellipse were designated as 'unique' samples for inclusion in the reference data set, as capturing this variance in a predictive model would most likely encompass future samples (Additional file 1: Figure S1). The remainder of the reference set was comprised of randomly selected plants that lay inside of the Hotelling $\mathrm{T}^{2}$ ellipse and were more representative of the sample majority.

\section{Spectral data acquisition}

MIR, NIR, and Raman spectra were collected for 245 diverse eucalypt and Acacia trees in 96-well plates. Figures 1, 2 , and 3 provide a spectral comparison between the three analytical methods. The spectral acquisition parameters (see Methods) were optimized to maximize the signal-tonoise $(\mathrm{S} / \mathrm{N})$. The tunable nature of the Raman excitation source is one of the main advantages to using Raman spectroscopy. It allows a two-pronged approach to $\mathrm{S} / \mathrm{N}$ optimization by amplifying the signal via higher excitation powers (although the noise can also increase), and decreasing the noise by augmenting the number of spectral scans. With the MIR and NIR instruments, the sole way to increase $\mathrm{S} / \mathrm{N}$ is by performing more scans of the data, which only reduces the noise. By intensifying the laser power, previously veiled spectral features can be elucidated, which ultimately can lead to more distinguished spectra. Multivariate models flourish when there is significant variance in the data set.

\section{Multivariate model assembly and evaluation}

The lignin S/G ratios quantified by pyMBMS (Additional file 1: Table S2) were linked with MIR, NIR, and Raman spectral data. The standard error of the laboratory (SEL) calculated for the pyMBMS reference data (0.05 to 0.06$)$ is listed in Tables 2 and 3, as well as Additional file 1: Table S2. This value represents the lowest possible predictive error a model can contain as a model cannot have lower error than the data used in its construction. Iterations of spectral processing techniques allowed the selection of those treatments that led to the most robust PLS models (Table 2). First and second derivative transformations were
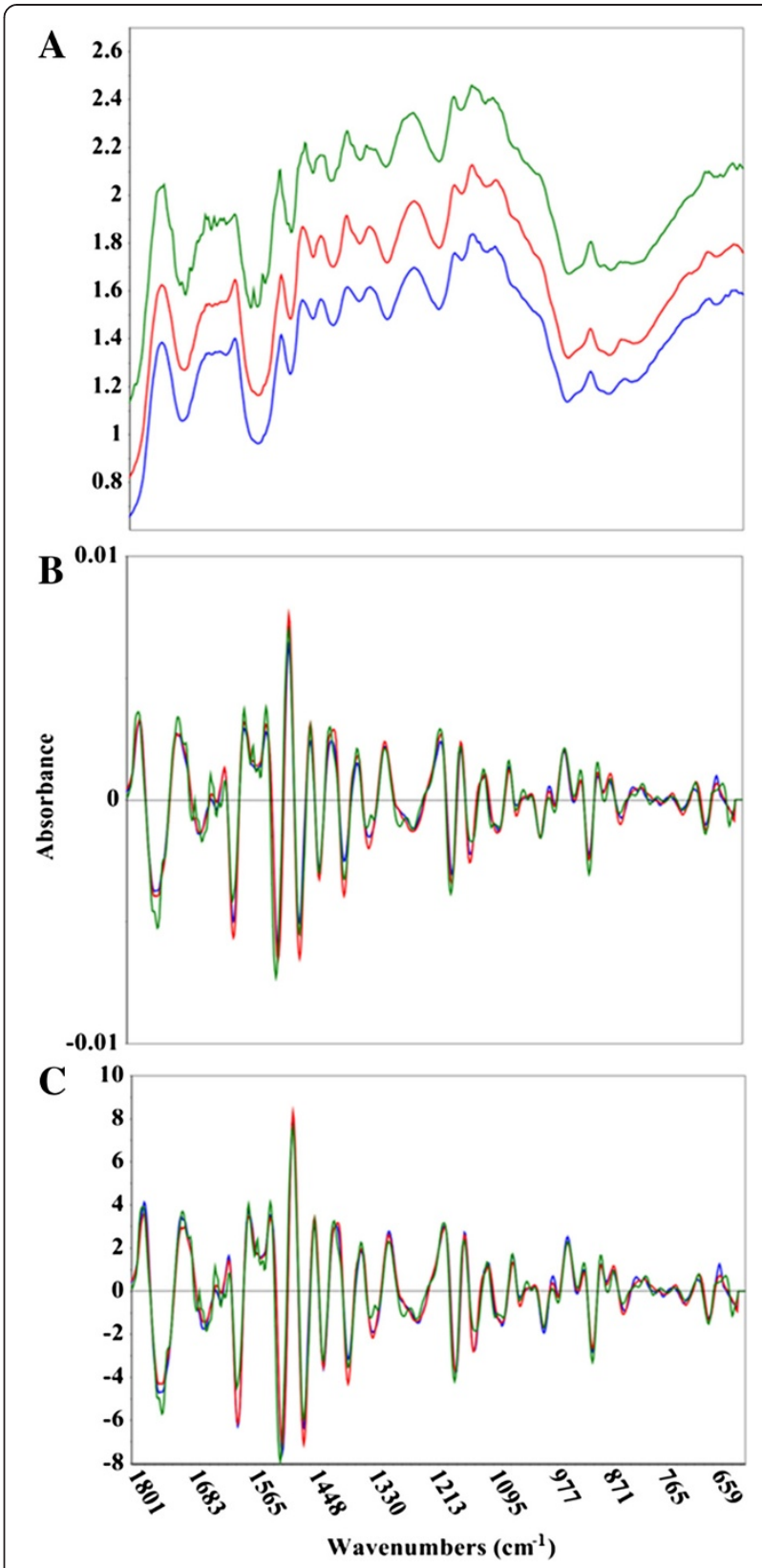

Figure 1 Comparison of raw and pretreated mid-infrared spectral data. Mid-infrared spectra of Acacia microbotrya (green), Corymbia hybrid (blue), and Eucalyptus globulus subspecies maidenii (red). The upper panel $(\mathbf{A})$ shows the untreated spectral data, while the middle (B) and bottom (C) panels show the second derivative, and second derivative + standard normal variate (SNV) spectral transformations, respectively. The $x$-axis is in wavenumbers while the $y$-axis is the absorbance.

employed to remove additive effects from the spectra, such as baseline offsets. Spectral smoothing was necessary to remove the additional spectral noise concomitant with performing derivative transformations. It is imperative that the spectra not be over-smoothed, as this can be deleterious to vital spectroscopic information. Multiplicative effects like 


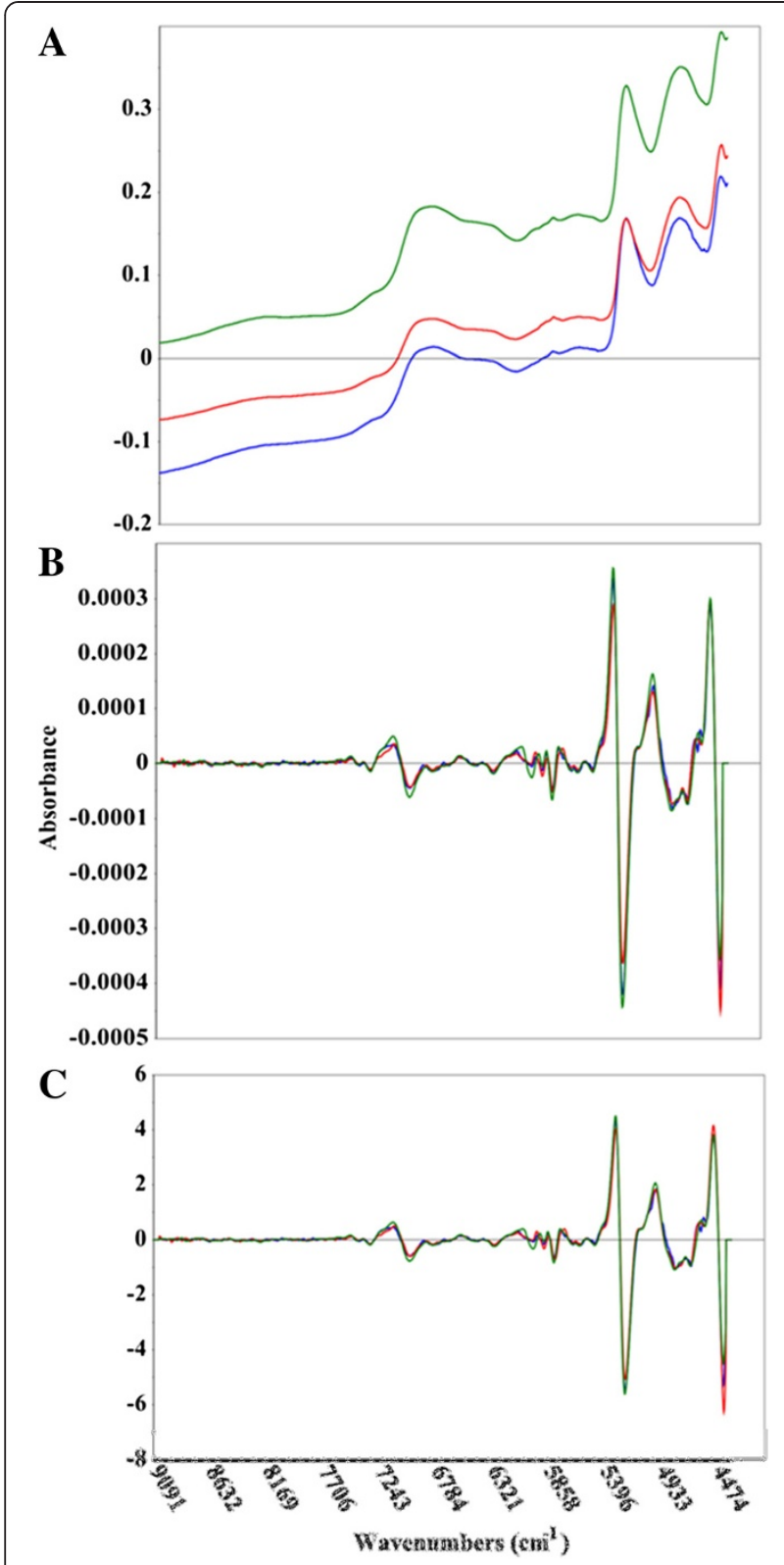

Figure 2 Comparison of raw and pretreated near-infrared spectral data. Near-infrared spectra of Acacia microbotrya (green), Corymbia hybrid (blue), and Eucalyptus globulus subspecies maidenii (red). The upper panel (A) shows the untreated spectral data, while the middle (B) and bottom (C) panels show the second derivative, and second derivative + standard normal variate (SNV) spectral transformations, respectively. The $\mathrm{x}$-axis is in wavenumbers while the $y$-axis is the absorbance.

variations in biomass particle size were removed using techniques such as standard normal variate (SNV), multiplicative scatter correction (MSC), or extended MSC (EMSC). Combinations of these spectral corrections were used to remove physical differences from the samples such that predictive models focused on chemical distinctions (second derivative + SNV).

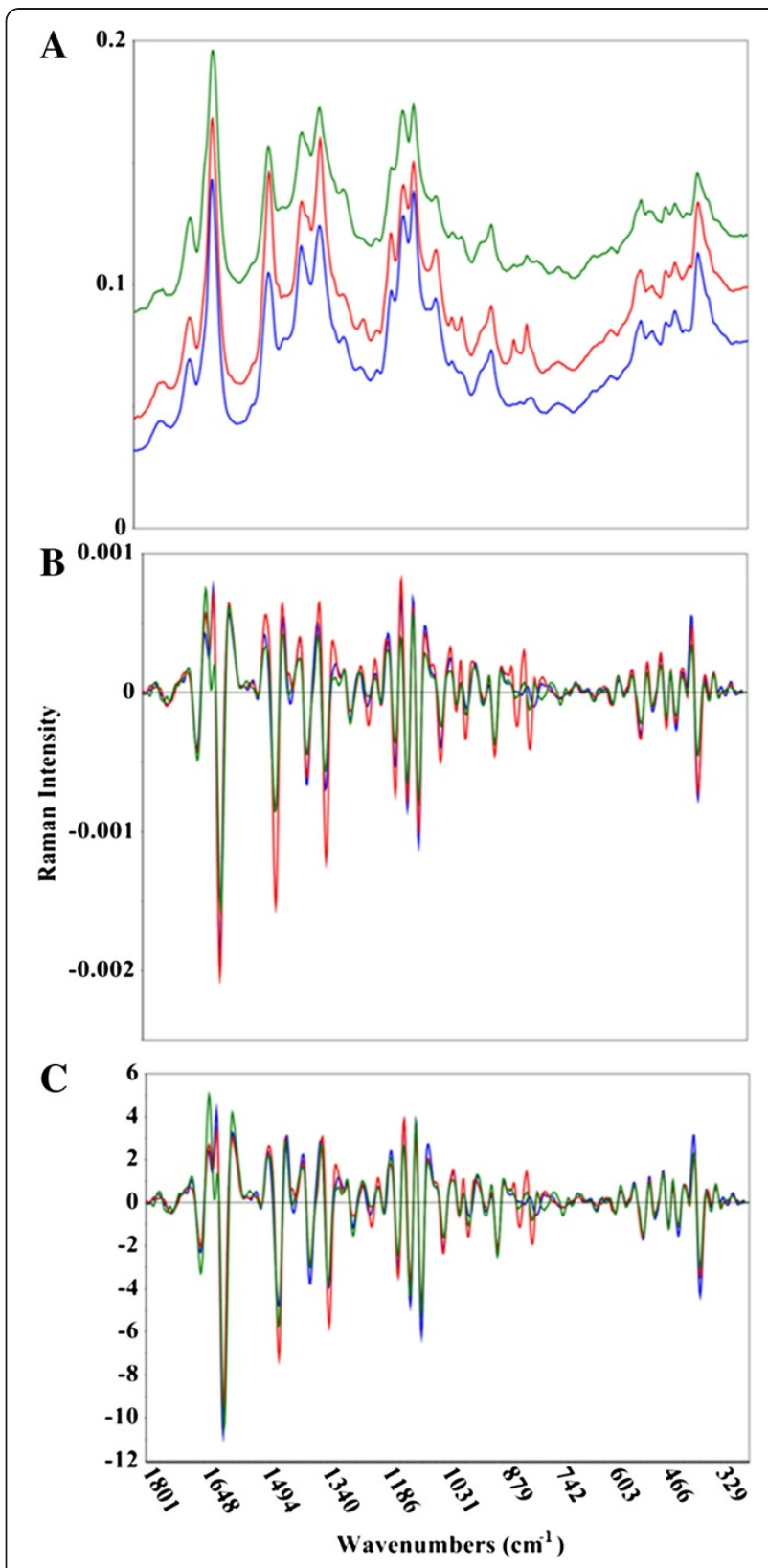

Figure 3 Comparison of raw and pretreated Raman spectral data.

Raman spectra of Acacia microbotrya (green), Conymbia hybrid (blue), and Eucalyptus globulus subspecies maidenii (red). The upper panel (A) shows the untreated spectral data, while the middle (B) and bottom (C) panels show the second derivative, and second derivative + standard normal variate (SNV) spectral transformations, respectively. The $x$-axis is in wavenumbers, while the $y$-axis shows the Raman intensity.

Tables 2 and 3 provide an assessment of the models .constructed using MIR, NIR, and Raman spectral data. The root mean standard error of cross-validation (RMSECV) is a measurement of the error in the predicted lignin $\mathrm{S} / \mathrm{G}$ ratio when cross-validation is employed to assess the models. Cross-validation is also called the 'leave-one-out' method, as one sample from 
Table 2 Comparison of PLS calibration models using vibrational spectroscopy and pyrolysis molecular beam mass spectrometry

\begin{tabular}{|c|c|c|c|c|c|c|c|}
\hline Method & SEL calibration ${ }^{1, a}$ & RMSEC $^{2, a}$ & RM- SECV 3,a & $R^{2} \mathrm{Cal}^{4}$ & $R^{2} \mathrm{CV}^{5}$ & $F^{6}$ & Outliers $^{7}$ \\
\hline Raman $2^{\text {nd }}$ deriv (19 pt) + SNV 32 scans & 0.05 & 0.13 & 0.14 & $0.83 \pm 0.02$ & $0.81 \pm 0.02$ & $4-5$ & 2 \\
\hline Raman $1^{\text {st }}$ deriv $(7 \mathrm{pt})+\mathrm{EMSC} 32$ scans & 0.05 & 0.13 & 0.14 & $0.845 \pm 0.003$ & $0.82 \pm 0.01$ & $4-5$ & 3 \\
\hline Raman EMSC $+2^{\text {nd }}$ deriv (15 pt) 96 scans & 0.05 & 0.13 & 0.14 & $0.83 \pm 0.01$ & $0.81 \pm 0.01$ & $5-6$ & $2-3$ \\
\hline Raman $2^{\text {nd }}$ deriv $(15 \mathrm{pt})+$ SNV 96 scans & 0.05 & 0.13 & 0.13 & $0.84 \pm 0.01$ & $0.82 \pm 0.01$ & $4-5$ & $4-5$ \\
\hline MIR EMSC $+2^{\text {nd }}$ deriv (15 pt) & 0.05 & 0.13 & 0.14 & $0.84 \pm 0.03$ & $0.81 \pm 0.03$ & $3-4$ & $1-2$ \\
\hline MIR $2^{\text {nd }}$ deriv $(17 p t)+$ MSC & 0.05 & 0.13 & 0.14 & $0.82 \pm 0.01$ & $0.78 \pm 0.01$ & $3-4$ & $1-2$ \\
\hline MIR $2^{\text {nd }}$ deriv $(17 \mathrm{pt})+\mathrm{SNV}$ & 0.05 & 0.13 & 0.14 & $0.85 \pm 0.02$ & $0.82 \pm 0.03$ & $3-4$ & $2-3$ \\
\hline NIR EMSC $+2^{\text {nd }}$ deriv (25 pt) & 0.05 & 0.17 & 0.18 & $0.73 \pm 0.01$ & $0.681 \pm 0.004$ & $4-5$ & $4-7$ \\
\hline NIR $2^{\text {nd }}$ deriv (25 pt) + MSC & 0.05 & 0.17 & 0.18 & $0.72 \pm 0.02$ & $0.68 \pm 0.02$ & $4-6$ & $1-5$ \\
\hline NIR $2^{\text {nd }}$ deriv $(25 \mathrm{pt})+\mathrm{SNV}$ & 0.05 & 0.16 & 0.17 & $0.74 \pm 0.01$ & $0.70 \pm 0.02$ & $4-5$ & $2-3$ \\
\hline
\end{tabular}

${ }^{1}$ Standard Error of the Laboratory for the calibration data.

${ }^{2}$ Root Mean Standard Error of Calibration.

${ }^{3}$ Root Mean Standard Error of Cross-Validation.

${ }^{4}$ Coefficient of determination for calibration set.

${ }^{5}$ Coefficient of determination for full cross-validation.

${ }^{6}$ Average number of factors used in model construction.

${ }^{7}$ Number of outliers removed from calibration models.

${ }^{a}$ Average errors of 3 randomly generated models using data provided. Models were not statistically different.

The numbers listed parenthetically reflect the degree of Savitzky-Golay spectral smoothing.

Statistical values are the average of 3 independent models.

MIR = mid-infrared spectroscopy, NIR = near-infrared spectroscopy, EMSC = extended multiplicative scatter correction, $\mathrm{MSC}=$ multiplicative scatter

correction, SNV = standard normal variate.

the data matrix is removed and subsequently predicted using the remaining samples. This technique proceeds for a set number of calibration samples (random CV) or is performed for all samples (full CV). While the RMSECV is a good indicator of a model's accuracy, a more aggressive way of evaluating PLS models is by using a randomly produced validation set, and monitoring the resultant RMSEP. This reflects the error that can be expected in the model when true predictions of unknown variables are attempted.

Table 3 Comparison of PLS predictive models using vibrational spectroscopy and pyrolysis molecular beam mass spectrometry

\begin{tabular}{|c|c|c|c|c|c|c|}
\hline Method & SEL validation $^{1, a}$ & SEP $^{2, a}$ & RMSEP $^{3, a}$ & $\mathrm{r}-\mathrm{Val}^{4}$ & $R^{2} \mathrm{Val}^{5}$ & Outliers $^{6}$ \\
\hline Raman $2^{\text {nd }}$ deriv (19 pt) + SNV 32 scans & 0.05 & 0.14 & 0.13 & $0.89 \pm 0.04$ & $0.79 \pm 0.08$ & 1 \\
\hline Raman $1^{\text {st }}$ deriv (7 pt) + EMSC 32 scans & 0.05 & 0.13 & 0.13 & $0.91 \pm 0.02$ & $0.83 \pm 0.04$ & 1 \\
\hline Raman EMSC $+2^{\text {nd }}$ deriv (15 pt) 96 scans & 0.05 & 0.14 & 0.15 & $0.90 \pm 0.02$ & $0.81 \pm 0.04$ & 0 \\
\hline Raman $2^{\text {nd }}$ deriv (15 pt) + SNV 96 scans & 0.06 & 0.17 & 0.16 & $0.86 \pm 0.02$ & $0.74 \pm 0.04$ & 0 \\
\hline MIR EMSC $+2^{\text {nd }}$ deriv (15 pt) & 0.05 & 0.14 & 0.13 & $0.87 \pm 0.06$ & $0.8 \pm 0.1$ & 1 \\
\hline MIR $2^{\text {nd }}$ deriv (17 pt) + MSC & 0.05 & 0.14 & 0.14 & $0.91 \pm 0.01$ & $0.83 \pm 0.01$ & 1 \\
\hline MIR $2^{\text {nd }}$ deriv (17 pt) + SNV & 0.05 & 0.15 & 0.15 & $0.87 \pm 0.02$ & $0.76 \pm 0.03$ & 1 \\
\hline NIR EMSC $+2^{\text {nd }}$ deriv (25 pt) & 0.06 & 0.19 & 0.20 & $0.79 \pm 0.01$ & $0.62 \pm 0.01$ & 0 \\
\hline NIR $2^{\text {nd }}$ deriv (25 pt) + MSC & 0.06 & 0.18 & 0.18 & $0.82 \pm 0.04$ & $0.67 \pm 0.07$ & 1 \\
\hline NIR $2^{\text {nd }}$ deriv $(25 \mathrm{pt})+\mathrm{SNV}$ & 0.06 & 0.22 & 0.21 & $0.80 \pm 0.04$ & $0.65 \pm 0.07$ & 1 \\
\hline
\end{tabular}

${ }^{1}$ Standard Error of the Laboratory for the validation data.

${ }^{2}$ Standard Error of Prediction.

${ }^{3}$ Root Mean Standard Error Prediction.

${ }^{4}$ Correlation coefficient for the validation set.

${ }^{5}$ Pearson coefficient of determination for validation.

${ }^{6}$ Number of outliers removed from validation models.

${ }^{a}$ Average errors of three randomly generated models using data provided. Models were not statistically different.

The numbers listed parenthetically reflect the degree of Savitzky-Golay spectral smoothing.

Statistical values are the average of 3 independent models.

MIR = mid-infrared spectroscopy, NIR = near-infrared spectroscopy, EMSC = extended multiplicative scatter correction, $M S C=$ multiplicative scatter correction, SNV = standard normal variate. 
Another metric that facilitates the development of robust multivariate models is the correlation coefficient $(r)$ or the coefficient of determination $\left(R^{2}\right)$. These parameters reflect the accuracy of the linear experimental trend line versus the ideal. Again, while probing these values for $\mathrm{CV}$ to isolate which spectral processing techniques result in the most robust, accurate models, assessing the $\mathrm{r}$ and $R^{2}$ magnitudes for the prediction of a true validation set permits a more rigorous analysis of model performance.

The number of factors used for model construction is also reported in Table 2. Over-fitting the data can result if more than the optimal number of factors is employed, as these superfluous factors attempt to explain random noise in the spectra. Adding and subtracting the number of factors used in the calculation of a model can diagnose overfitting of the data. For example, using more factors than is optimal may result in seemingly more accurate calibration models, but will result in more erroneous validation and prediction. Evaluation of residual variance plots or Scree plots (Additional file 1: Figure S2) aided in determining the appropriate number of factors to use for the model. Another technique to assess over-fitting is by gauging the validation metrics, namely the $R^{2}$ and RMSEP as these parameters will be significantly less accurate from those obtained using cross-validation if over-fitting has occurred.

Predictive models comprised of MIR spectra resulted in a RMSEC average of 0.13 regardless of the spectral transformations selected, and calibration $R^{2}$ values between $0.82 \pm 0.01$ and $0.85 \pm 0.02$ (Table 2, and Additional file 1: Table S3). The models used either three or four factors to successfully explain the maximum amount of variance, without over-fitting the data. Of the 195 calibration samples, only two were characterized as outliers after thoroughly evaluating the leverage, Hotelling $\mathrm{T}^{2}$, residual variance, and X-Y distribution statistical plots, although this characterization was dependent on the randomized calibration and validation matrices. Thus, one of the two samples could occasionally be left in a data matrix without detrimental effects on the model. The RMSEP averages, using MIR spectroscopy were 0.13 to 0.15 , with $r$ values between $0.87 \pm 0.02$ and $0.91 \pm 0.01 \quad\left(R^{2}=0.76 \pm 0.03\right.$ to $0.83 \pm 0.01$, Table 3 , Additional file 1: Table S3). Figure 4A demonstrates the suitability of using MIR spectroscopy to forecast lignin S/G ratios. The plot represents the best MIR model using second derivative + MSC-transformed spectral data with the pyMBMS results. The experimental trend line (blue) closely resembles the target line (black), indicating high correlation.

NIR spectroscopy, although used quite frequently in developing successful multivariate models, exhibited the highest RMSEC (0.16 to 0.17 ) and RMSEP (0.18 to 0.21 ), and lowest calibration $R^{2}(0.72 \pm 0.02$ to $0.74 \pm 0.01)$ and validation $r$ values $\left(0.79 \pm 0.01\right.$ to $0.82 \pm 0.04, R^{2}=0.62 \pm 0.01$ to $0.67 \pm 0.07$ ) (Tables 2 and 3, Additional file 1: Table S3).
The NIR spectral data required four to six factors to explain the variance and had between one and five samples characterized as an outlier, depending on the calibration and validation matrices. Samples lacking in predictive accuracy, whether in full cross-validation or randomized validation models, were left in the model unless distinguished as outliers using the aforementioned plots. Figure 4B shows the predicted versus reference plot using second derivative + MSC-transformed spectral data. The experimental trend line (blue) shows significant deviation from the target line (black).

The Raman spectral data produced PLS models with a RMSEC average of 0.13 , regardless of the spectral transformations selected (Table 2, Additional file 1: Table S3). The calibration $R^{2}$ values ranged from $0.83 \pm 0.01$ to $0.845 \pm$ 0.003 , four to six factors were required to explain the variance, and between two and five samples were shown to be outliers, dependent on the respective calibration and validation matrices (Table 2, Additional file 1: Table S3). The RMSEP averages ranged from 0.13 to 0.16 , with $r$ values between $0.86 \pm 0.02$ and $0.91 \pm 0.02\left(R^{2}=0.74 \pm 0.04\right.$ to $0.83 \pm 0.04$, Table 3, Additional file 1: Table S3). Figure 4C illustrates the competency of Raman spectroscopy to accurately predict lignin S/G ratios. In this specific model, first derivative + EMSC-transformed spectral data was coupled with the corresponding pyMBMS results. As with the MIR model (Figure 4A), the experimental trend line (blue) shows nearly superimposable correlation with the target line (black).

One of the preliminary steps in constructing a multivariate model is the mean-centering of the spectral data. An average spectrum is determined and subtracted from each individual spectrum, leaving the residual variance from the mean. It is this variance that multivariate methods splice with reference results. Figures 1, 2, and 3 show representative MIR, NIR, and Raman spectral data. The spectra have been truncated to show the regions used in the construction of the models, but have not been otherwise offset or modified. The raw Raman spectra contain substantial differences in measured vibrational modes as well as spectral intensities (Figure 3A). This is exemplified by considering the $\mathrm{C}-\mathrm{C}$ stretching vibration of the polysaccharides near $1100 \mathrm{~cm}^{-1}$, the lignin $\mathrm{C}-\mathrm{C}-\mathrm{H}$ and $-\mathrm{HC}=\mathrm{CH}$ deformation at $973 \mathrm{~cm}^{-1}$, and the $\mathrm{C}-\mathrm{O}$ stretch, aryl symmetric bend, and $\mathrm{C}-\mathrm{H}$ out of plane bend at $808 \mathrm{~cm}^{-1}[35,36]$. The plot of raw MIR spectra reveals greater similarities between the spectra, although there remain some spectral differences, such as the aromatic skeletal vibration at $1595 \mathrm{~cm}^{-1}$ (Figure 1A). Spectral differences in both the Raman and MIR spectra are further elucidated when the second derivative transformation has been applied (Figures $1 \mathrm{~B}$ and $3 \mathrm{~B}$ ). The NIR spectra display considerable resemblance, and the lack of distinguished spectral features makes it challenging to visually differentiate 

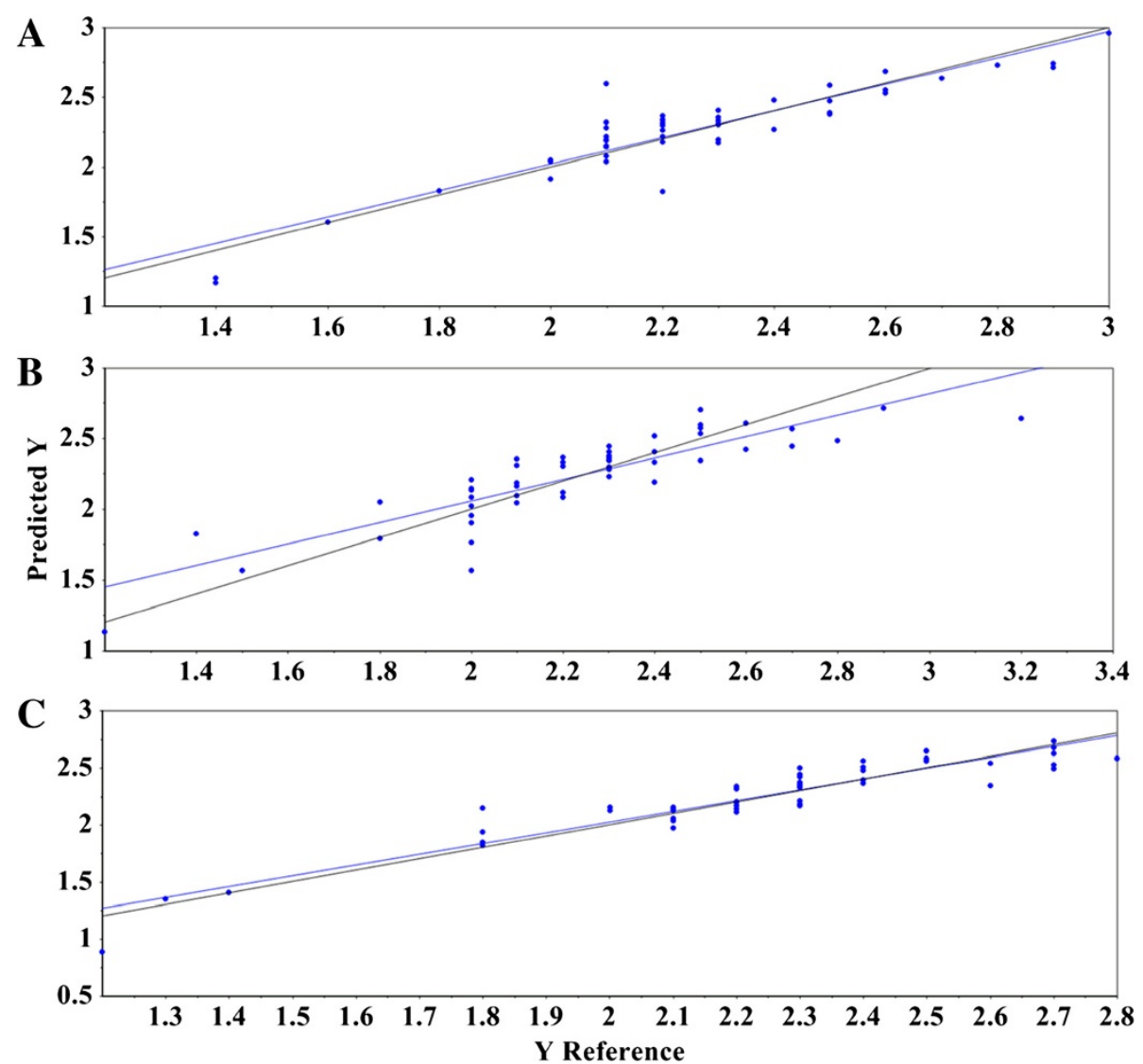

Figure 4 Reference versus predicted plot for validation set using mid-infrared, near-infrared, and Raman spectral data. (A) Plot of the predicted lignin S/G ratio using a model built from second derivative + MSC-transformed mid-infrared spectra and the reference pyMBMS data. The black line indicates the target line of optimal fit and the blue line represents the experimental fit of the data to the model. (B) Plot of the predicted lignin S/G ratio using a model built from second derivative + MSC-transformed near-infrared spectra and the reference pyMBMS data. The black line indicates the target line of optimal fit and the blue line represents the experimental fit of the data to the model. (C) Plot of the predicted lignin S/G ratio using a model built from first derivative + EMSC-transformed Raman spectra and the reference pyMBMS data. The black line indicates the target line of optimal fit and the blue line represents the experimental fit of the data to the model. The $\mathrm{x}$-axis shows the pyMBMS measured lignin S/G ratio, and the $y$-axis reveals the predicted lignin S/G ratios. S/G = syringyl-to-guaiacyl ratio, MSC=multiplicative scatter correction, pyMBMS = pyrolysis molecular beam mass spectrometry, EMSC = extended multiplicative scatter correction.

between the samples (Figure 2). The second derivative NIR spectra (Figure 2B) reveal relatively few chemical signatures, especially when juxtaposed to both Raman and MIR spectral data. The accuracy of the MIR and Raman predictive models is likely correlated with the higher abundance of both spectral detail and variation.

The analysis of regression coefficient plots enables the determination of which spectral regions are deemed integral to model construction. Tables 4, 5, and 6 list the spectral regions identified by the regression coefficients plots for MIR, Raman, and NIR models, respectively, while Additional file 1: Figures S3, S4, and S5 illustrate examples of these plots graphically. The regression coefficient wavenumber regions listed encompass the range of vibrational modes used in calculating the models, but does not signify that the complete range was utilized. Tables 4, 5, and 6 demonstrate that the models successfully extracted key lignin and lignin model compound vibrational modes. Although there is overlap between some of the spectral assignments, there is general agreement between the sources as to peak location, and their classification as bonds representative of lignin and lignin monomers. It should be noted that different instrumental configurations can lead to variation in vibrational mode peak locations.

The most intense vibrational modes of cellulose occur at 1091 and $1117 \mathrm{~cm}^{-1}$ [36]. While these, and less intense peaks indicative of cellulose, are encompassed in the spectral regions identified by the MIR and Raman regression coefficients plots, further analysis revealed that the known cellulose or polysaccharide vibrational modes (such as $1091 \mathrm{~cm}^{-1}$ ), were negatively correlated (for example, Additional file 1: Figure S4) or were not identified as important to the model construction (896, 1074, and $1268 \mathrm{~cm}^{-1}$ ). A positive correlation was 
Table 4 MIR vibrational mode regions identified from regression coefficient plots and spectral assignments corresponding to lignin and/or lignin monomers

\begin{tabular}{|c|c|}
\hline $\begin{array}{l}\text { Vibrational mode from } \\
\text { regression coefficients plot }\end{array}$ & $\begin{array}{l}\mathrm{S} / \mathrm{G} / \mathrm{H} \text { vibrational mode } \\
\text { and spectral assignment }\end{array}$ \\
\hline 788-790 & $784(\mathrm{G})[37]$ \\
\hline \multirow[t]{2}{*}{ 808-836 } & $813(\mathrm{G})[37]$ \\
\hline & $827(S)[38]$ \\
\hline 854-883 & $863,878(G)[37]$ \\
\hline 912-917 & $914(\mathrm{G})[37]$ \\
\hline \multirow[t]{2}{*}{$1137-1168$} & $1142(G)[39]$ \\
\hline & $1151(G)[40]$ \\
\hline \multirow[t]{2}{*}{$1205-1263$} & 1215 (Lignin) [40] \\
\hline & $1226(G), 1252$ [39] \\
\hline \multirow[t]{2}{*}{ 1270-1299 } & $1270(\mathrm{G})[39]$ \\
\hline & $1269(\mathrm{G})[40]$ \\
\hline \multirow[t]{3}{*}{$1319-1425$} & 1425 (S) [38] \\
\hline & 1330, 1425 (S), 1379, 1428 (Lignin) [39 \\
\hline & $1327(\mathrm{G}), 1425,1427[40]$ \\
\hline \multirow[t]{3}{*}{$1442-1502$} & 1500 (S) [38] \\
\hline & 1465 (Lignin) [39] \\
\hline & $1462,1463[40]$ \\
\hline \multirow[t]{2}{*}{$1508-1521$} & 1506-1513 [39] \\
\hline & $1513,1514[40]$ \\
\hline \multirow[t]{3}{*}{ 1585-1606 } & 1589 (S) [38] \\
\hline & 1596-1600 (Lignin) [39] \\
\hline & $1594,1603[40]$ \\
\hline 1610-1612 & 1610 [41] \\
\hline $1698-1714$ & $1704[40]$ \\
\hline $1745-1756$ & $1733-1753[42]$ \\
\hline
\end{tabular}

$\mathrm{G}=$ guaiacyl, $\mathrm{S}=$ syringyl.

determined for peaks due to cellulose and lignin vibrational modes (for example, 1117 and $1338 \mathrm{~cm}^{-1}$ ). The NIR regression coefficients plots contained various regions that were not attributable to lignin and lignin derived molecules besides those itemized in Table 6. Given the limited spectral diversity measured using NIR, since only overtone and combination bands are evaluated, interpreting regression coefficients plots becomes more ambiguous regarding what is being predicted.

To summarize, the RMSEP values measured in MIR and Raman models (0.13 to 0.16 , approximately two to three times higher than the SEL) did not statistically differ, indicative that either spectroscopic technique could be used to obtain similar results. The NIR models resulted in a RMSEP of 0.18 to 0.21 (three to four times higher than the SEL. This increase in error may not warrant migration from using NIR spectroscopy as an analytical tool for developing predictive multivariate models however, especially when factors such as instrument expense and ease of use are considered. Ultimately, identifying other useful applications of the MIR and Raman instrumentation (for example, structural analysis) will aid in selecting which analytical tool(s) will be appropriate.

The models produced in this study contain 17 different plant species across three genera. Rather than assemble three separate models for each genus, a more global approach was undertaken to construct one robust model capable of predicting both heartwood and sapwood samples from Acacia, Corymbia, and Eucalyptus. Additionally, the metrics listed in Tables 2 and 3 are the average results obtained from using three randomized calibration and validation matrices for each spectral transformation (Additional file 1: Table S3). This tactic permitted the development of completely independent predictive models, whereas the use of one set of calibration and validation matrices for all spectroscopic data sets may have introduced a level of undesirable bias into the models.

\section{Conclusions}

Eucalypts, including Corymbia and Eucalyptus, and Acacias present an attractive option for biofuel and biochemical production. Given the pool of over 900 diverse species of eucalypts, it is essential to isolate biomass species possessing traits found to play important roles in diminishing biomass recalcitrance. The standard methods of analysis are time-consuming, potentially toxic, and can destroy the sample. The use of multivariate modeling can significantly reduce experiment and analysis time and expense. This research has illustrated the validity of vibrational spectroscopy to provide non-destructive, accurate, global, predictive models encompassing an assorted array of feedstocks for the determination of lignin S/G ratios. Models constructed using MIR and Raman spectral data resulted in more accurate predictions compared to those produced from NIR spectra (RMSEP of 0.13 to 0.16 for MIR and Raman versus 0.18 to 0.21 for NIR). Current investigations are also underway to apply vibrational spectroscopy and PLS modeling for the prediction of cell-wall composition, including cellulose, xylan, and lignin content, and projected glucose and xylose release.

\section{Methods}

\section{Wood samples}

The 245 wood samples were obtained from a range of subtropical and temperate tree species either currently used for pulp or timber plantations or were prospective species for biomass and/or oil production [34]. The trees studied were as follows: Corymbia citriodora subspecies citriodora, Corymbia torelliana, Corymbia citriodora subspecies variegata, several families of Corymbia hybrids [50], Eucalyptus argophloia, Eucalyptus cloeziana, Eucalyptus crebra, Eucalyptus dunnii, Eucalyptus globulus subsp. maidenii, 
Table 5 Raman vibrational modes identified from regression coefficient plots and spectral assignments corresponding to lignin and/or lignin monomers

\begin{tabular}{lc}
\hline $\begin{array}{l}\text { Vibrational mode from } \\
\text { regression coefficients plot }\end{array}$ & $\begin{array}{c}\text { S/G/H vibrational mode } \\
\text { and spectral assignment(s) }\end{array}$ \\
\hline $351-376$ & $369(\mathrm{~S}), 357,370(\mathrm{G})[35]$ \\
$378-401$ & $370-399(\mathrm{~S})[20]$ \\
$474-623$ & $529,564,582(\mathrm{~S}), 541,559,590(\mathrm{G})[35]$ \\
$665-725$ & $711(\mathrm{~S})[43]$ \\
$736-756$ & $712(\mathrm{G}), 701(\mathrm{H})[35]$ \\
& $741(\mathrm{~S})[43]$ \\
$748-765$ & $741(\mathrm{H})[35]$ \\
$765-796$ & $761(\mathrm{G})[43]$ \\
& $781-820(\mathrm{~S})[20]$ \\
& $784(\mathrm{G})[43]$ \\
& $793(\mathrm{G})[35]$
\end{tabular}

$800-835$

$875-939$

991-1051

$1091-1131$

1135-1195

$1205-1242$

$1261-1346$

$1434-1448$

1587-1606

$$
\text { 819-864 (H) [20] }
$$$$
810 \text { (S) [43] }
$$$$
799(\mathrm{~S}), 823(\mathrm{H})[35]
$$$$
920(\mathrm{G}) \text { [43] }
$$$$
907(\mathrm{~S}), 921(\mathrm{G}) \text { [35] }
$$$$
1024(G)[43]
$$

$1108(\mathrm{~S}), 1124(\mathrm{G}), 1094(\mathrm{H})[43]$

$1116(\mathrm{~S}), 1122(\mathrm{G}), 1105(\mathrm{H})[35]$

$$
\begin{gathered}
1154(\mathrm{~S}), 1158(\mathrm{G}), 1168(\mathrm{H}), \\
1170 \text { (Lignin) [28] }
\end{gathered}
$$

$1138-1160(\mathrm{~S}), 1162-1188(\mathrm{G})$, 1163-1179 (H) [20]

$1148(\mathrm{~S}), 1186(\mathrm{G}), 1164(\mathrm{H})[43]$

$1152,1187(\mathrm{~S}), 1155,1186(\mathrm{G})$, $1173,1199(\mathrm{H})[35]$

$1200(H)[28]$

1213-1218 (H) [20]

$1228(\mathrm{~S}), 1215(\mathrm{H})[43]$

1214, 1241 (S), 1208, 1241 (G), $1216(H)$ [35]

1337 (S), $1263(\mathrm{H}), 1270$ (Lignin) [28]

1262-1275 (G), 1318-1332, 1331-1338

(S), 1286-1299 (H) [20]

1331 (S), 1270-1285 (G), 1338 H [43]

1331 (S), 1272, 1288 (G), 1298, 1331 (H) [35]

$1454-1460(\mathrm{~S}), 1452-1465(\mathrm{G})$

$1452(\mathrm{~S}), 1455(\mathrm{G}), 1455(\mathrm{H})$ [35]

$1594(S), 1589,1604(G), 1588,1606(H)$, 1591, 1604 (Lignin) [28]

1588 (S) [43]
$1043(\mathrm{~S}), 1036$ (G) [35]
Table 5 Raman vibrational modes identified from regression coefficient plots and spectral assignments corresponding to lignin and/or lignin monomers (Continued)

\begin{tabular}{lc}
\hline $1609(\mathrm{~S}), 1609(\mathrm{G}), 1599(\mathrm{H})[35]$ \\
$1623-1629$ & $1634(\mathrm{~S}), 1633(\mathrm{G}), 1632(\mathrm{H})$, \\
$1634(\mathrm{Lignin})[28]$ & coniferyl (G) and sinapyl (G) alcohol [44] \\
\hline G = guaiacyl, $\mathrm{S}=$ syringyl, $\mathrm{H}=$ = p-coumaryl.
\end{tabular}

Eucalyptus grandis, Eucalyptus kochii, Eucalyptus longirostrata, Eucalyptus loxophleba, Eucalyptus moluccana, Eucalyptus polybractea, Acacia microbotrya, and Acacia saligna. Trees were selected from trial sites in New South Wales (1), Western Australia (3) or Queensland (4) (Additional file 1: Table S1). Trees from New South Wales and Queensland sites were from Queensland Department of Agriculture, Fisheries and Forestry trials. They were sampled around 10 years of age except for the Narayan site (Additional file 1: Table S1) which was sampled at age 19 years. The trees chosen for this study stratified the size classes of the species in the trials and the samples were collected from 10 to 15 trees per species per site to ensure the full species diversity for wood property traits was captured. Approximately 2 to $3 \mathrm{~g}$ of drill swarf was obtained by drilling a $4 \mathrm{~cm}$ hole in the main stem at breast height (between 1.2 and $1.4 \mathrm{~m}$ height) in each tree using a $16 \mathrm{~mm}$ spade bit on a battery powered drill, after removing the outer-bark. Swarf was collected into paper bags and allowed to dry to ambient moisture levels in an air conditioned room for at least 48 hours prior to transfer into plastic vials $(10 \mathrm{ml}$ Falcon tubes) for storage and shipment. Similar sampling techniques were used for the material from Western Australia. All samples were ground for five minutes using the Joint BioEnergy Institute (Sandia National Laboratories) Biomass Preparation System robot created at Labman

Table 6 NIR vibrational modes identified from regression coefficient plots and spectral assignments corresponding to lignin and/or lignin monomers

\begin{tabular}{lc}
\hline $\begin{array}{l}\text { Vibrational mode from } \\
\text { regression coefficients plot }\end{array}$ & Lignin vibrational mode \\
\hline $4400-4586$ & $4411[45]$ \\
& $4546[46]$ \\
& $4686[47]$ \\
$5581-5600$ & $5583[45]$ \\
$5959-6009$ & $5963,5978[45]$ \\
$7081-7197$ & $5974,5978,5980[46]$ \\
$8459-8674$ & $7092[48]$ \\
$8720-8801$ & $8547[46]$ \\
\hline
\end{tabular}


Automation Ltd. (North Yorkshire, UK). The samples were oven-dried at $105^{\circ} \mathrm{C}$ for approximately 30 minutes to decrease moisture content before the spectra were collected.

\section{Fourier-transform infrared spectroscopy}

MIR spectra of raw biomass were collected with a Bruker Vertex 70 (Billerica, Massachusetts, United States) spectrometer, equipped with a HTS-XT 96-well microplate reader (Bruker, Billerica, Massachusetts, United States). Each spectrum was acquired in absorbance mode using 96 scans and a spectral resolution of $4 \mathrm{~cm}^{-1}$. Three representative spectra were averaged for each sample using the Bruker OPUS software package (Billerica, Massachusetts, United States). All other spectral processing was performed using the Unscrambler X (Camo Inc., Oslo, Norway). The MIR spectra (Figure 1) were collected using 96 scans, as more scans did not lead to a visually significant reduction of noisy spectral regions (data not shown). There is a tradeoff between the number of scans and the amount of time spent acquiring the spectra. A 96-well plate could be measured in approximately 48 minutes (30 seconds per sample) using 96 scans per sample.

\section{Near-infrared spectroscopy}

NIR spectra of raw biomass were collected using a Bruker FT-NIR Multi-Purpose Analyzer (Billerica, Massachusetts, United States) in diffuse reflectance mode, also equipped with the HTS-XT 96-well microplate reader. Each spectrum was acquired using 256 scans and a spectral resolution of $8 \mathrm{~cm}^{-1}$. Three representative spectra were averaged for each sample using the Bruker OPUS software package. All other spectral processing was performed using the Unscrambler X. The NIR spectra (Figure 2) were collected using 256 scans, since the signal was intrinsically much weaker than MIR spectral intensities due to the excitation of combination and overtone, rather than fundamental vibrational modes. Additionally, the use of the 96-well plate resulted in lower spectral intensities compared to measuring the samples individually in glass vials. Using 256 scans per sample, a accept plate could be measured with NIR spectroscopy in approximately one hour.

\section{Fourier-transform Raman spectroscopy}

Raman spectra (Figure 3) of raw biomass were collected with a Bruker MultiRAM Stand Alone Spectrometer (Billerica, Massachusetts, United States) equipped with a HTS-mapping stage for measuring samples in glassbottomed 96-well plates. The $1064 \mathrm{~nm}$ tunable Nd:YAG laser was programmed to $350 \mathrm{~mW}$ to maximize the Raman scatter. Each spectrum was acquired using 32 and 96 scans, with a spectral resolution of $4 \mathrm{~cm}^{-1}$. Three representative spectra were averaged for each sample using the Bruker OPUS software package. All other spectral processing was performed using the Unscrambler X. The total analysis time was kept at approximately one hour by employing 32 spectral scans. An additional data set using 96 scans was collected to provide a more direct comparison between MIR and Raman spectra. A 96-well plate could be measured in about five hours using 96 scans (about three minutes per sample).

\section{Reference data set}

The spectral pre-processing for PCA included baseline correction and either seven- (NIR) or nine-point (MIR) Savitzky-Golay smoothing. The resultant PCA allowed the rough classification of 'unique' plant samples, as determined by using the scores, Hotelling $\mathrm{T}^{2}$, leverage, and residual variance plots. The reference data set was composed of the 'unique' plants established from the PCA metrics, and a random selection of feedstocks representative of the majority of the samples. The spectral regions used for PCA analysis were 8794 to $3999 \mathrm{~cm}^{-1}$ for NIR and 5303 to $599 \mathrm{~cm}^{-1}$ for MIR.

\section{Pyrolysis/molecular beam mass spectrometry}

A custom built pyrolysis/molecular beam mass spectrometer (pyMBMS), at the National Renewable Energy Lab (Golden, Colorado, USA), was used as the reference technique for quantifying $S$ and $G$ lignin ratios. The instrumental details have been previously reported [4]. A full description is available in Additional file 1. The peaks representative of lignin were those with mass-to-charge ratio $(\mathrm{m} / \mathrm{z})=120,124,137,138,150,152,154,164,167,178$, $180,181,182,194$, and 210. S/G ratios were calculated by dividing the sum of the syringyl peaks at 154, 167, 168, $182,194,208$, and 210 by the sum of guaiacyl peaks at $124,137,138,150,164$, and 178 . It is worth noting that a few lignin peaks had correlations with both $S$ and $G$ precursors, and subsequently were left out of the $S$ and $G$ calculations.

\section{Multivariate analysis}

The spectral data (MIR, NIR, and Raman, Figures 1, 2, and 3) was imported from OPUS into the Unscrambler X. A variety of spectral processing techniques were employed to see which methods appropriately corrected for additive and multiplicative effects, such as baseline offsets and particle size variations. The spectral transformations included: first or second Savitzky-Golay derivatives, standard normal variate (SNV), multiplicative scatter correction (MSC), and extended MSC (EMSC), as well as combinations of a derivative and SNV, MSC, or EMSC. Since the derivative transformations increase spectral noise, iterations of varying degrees of spectral smoothing were tested to see which reduced noise without sacrificing signal.

Reference sets of 195 randomly selected samples were used for PLS model construction. Each model was built 
using transformed spectral data that had been meancentered. The regression coefficients plot was used to identify which spectral variables were paramount in constructing the model, and recalculation of each model by using the marked important variables removed featureless spectral regions, thereby reducing spectral noise in the model. A full cross-validation was used to evaluate how the spectral transformations affected model accuracy. Validation sets of 50 randomly selected reference samples (separate from the 195 calibration samples) were used to evaluate the accuracy and robustness of the model's predictive capacity. The randomization was achieved in Microsoft Excel, using the random number generator function. This was performed three times for each method of spectroscopic processing. For example, three randomly generated calibration and validation matrices were combined with the Raman spectral data that had been transformed using a first derivative and EMSC. As shown in Additional file 1: Table S3, the models created using this procedure were not statistically different.

\section{Additional file}

Additional file 1: Table S1. Environmental characteristics for the plant growing sites. Table S2. Lignin S/G ratios as determined by pyrolysis molecular beam mass spectrometry. Table S3. Individual model parameters. Figure S1. MIR (top) and NIR (bottom) scores plots used to determine 'unique' samples. Figure S2. Example of residual variance or Scree plot used in determining the appropriate number of factors. Figure S3. Example of MIR regression coefficient plots used to determine which spectral regions used to construct the models. Figure S4. Example of Raman regression coefficient plots used to determine which spectral regions used to construct the models. Figure S5. Example of NIR regression coefficient plots used to determine which spectral regions used to construct the models.

\section{Abbreviations}

EMSC: Extended Multiplicative Scatter Correction; GC/MS: Gas Chromatography/ Mass Spectrometry; MIR: Mid-infrared Spectroscopy; MSC: Multiplicative Scatter Correction; NIR: Near-infrared Spectroscopy; PCA: Principal Component Analysis; PLS: Partial Least Squares Regression; pyMBMS: Pyrolysis Molecular Beam Mass Spectrometry; r: Coefficient of Correlation for Validation Set; $R^{2} C a l$ : Coefficient of Determination for Calibration Set; $R^{2} C V$ : Coefficient of Determination for Full Cross-Validation; $R^{2}$ Val: Coefficient of Determination for Validation Set; RMSEC: Root Mean Standard Error of Calibration; RMSECV: Root Mean Standard Error of Cross-Validation; RMSEP: Root Mean Standard Error of Prediction; SEL: Standard Error of the Laboratory; SEP: Standard Error of Prediction; SNV: Standard Normal Variate.

\section{Competing interests}

The authors declare that they have no competing interests.

\section{Authors' contributions}

JSL designed all spectroscopic methodology, collected the spectral data, created the multivariate analysis models, and wrote the manuscript. SS and BAS provided guidance throughout all experimentation and critically revised the manuscript. MD provided assistance with the multivariate analysis. DL and MS selected and collected the majority of the wood samples for analysis and provided information regarding the environmental and growing conditions. $\mathrm{RH}$ conceived the project, provided the funding, and critically revised the manuscript. All authors read and approved the final manuscript.

\section{Acknowledgements}

This manuscript was supported as part of a collaboration between the Queensland Alliance for Agriculture and Food Innovation and the Joint BioEnergy Institute. The work conducted by the Joint BioEnergy Institute was supported by the Office of Science, Office of Biological and Environmental Research, of the US Department of Energy under contract number DE-ACO2-05CH11231. The authors would like to thank Robert Sykes and Erica Gjersing at the National Renewable Energy Lab for obtaining the pyMBMS data and providing assistance and guidance with respect to the high-throughput pyMBMS pipeline, John Bartle at the Western Australian Department of Environment and Conservation, and Adam Healey at the University of Queensland, for the collecting and processing of some the wood samples and for information regarding the environmental specifications of the growing sites. The material from the Queensland and New South Wales sites was accessed from Queensland Department of Agriculture, Fisheries and Forestry trials.

\section{Author details}

${ }^{1}$ Queensland Alliance for Agriculture and Food Innovation, University of Queensland, 306 Carmody Road, St. Lucia, QLD 4072, Australia. ${ }^{2}$ Joint BioEnergy Institute, Lawrence Berkeley National Laboratory, 5885 Hollis Street, Emeryville, CA 94608, USA. 'Biological and Materials Science Center, Sandia National Laboratories, 7011 East Avenue, Livermore, CA 94551, USA. ${ }^{4}$ BioEnergy Science Center, Oak Ridge National Laboratory, 1 Bethel Valley Rd, Oak Ridge, TN 37831, USA. ${ }^{5}$ National Bioenergy Center, National Renewable Energy Laboratory, 15013 Denver West Parkway, Golden, CO 80401, USA. ${ }^{6}$ Forest Industries Research Centre, University of the Sunshine Coast and Queensland Department of Agriculture, Fisheries and Forestry, Locked Bag 4, Maroochydore DC, QLD 4558, Australia. ${ }^{7}$ Southern Cross Plant Science, Southern Cross University, Military Road, East Lismore, NSW 2480, Australia.

Received: 18 March 2014 Accepted: 28 May 2014

Published: 17 June 2014

\section{References}

1. Henry RJ: Evaluation of plant biomass resources available for replacement of fossil oil. Plant Biotechnol J 2010, 8:288-293.

2. Karp A, Shield I: Bioenergy from plants and the sustainable yield challenge. New Phytol 2008, 179:15-32.

3. Sims REH, Mabee W, Saddler JN, Taylor M: An overview of second generation biofuel technologies. Bioresour Technol 2010, 101:1570-1580.

4. Sykes R, Yung M, Novaes E, Kirst M, Peter G, Davis M: High-throughput screening of plant cell-wall composition using pyrolysis molecular beam mass spectroscopy. Methods Mol Bio 2009, 581:169-183.

5. Browning BL: Wood Lignins. In The Chemistry of Wood. Edited by Browning BL. New York, NY: Interscience; 1963:249-311.

6. Sarkanen KV, Hergert HL: Classification and Distribution. In Lignins: Occurrence and Formation, Structure, Chemical and Macromolecular Properties, and Utilization. Edited by Sarkanen KV, Ludwig CH. New York, NY: John Wiley \& Sons; 1971:43-94.

7. Davison BH, Drescher SR, Tuskan GA, Davis MF, Nghiem NP: Variation of S/G ratio and lignin content in a Populus family influences the release of xylose by dilute acid hydrolysis. Appl Biochem Biotechnol 2006, 129-132:427-435.

8. Tsutsumi Y, Kondo R, Sakai K, Imamura H: The difference of reactivity between syringyl lignin and guaiacyl lignin in alkaline systems. Holzforschung 1995, 49:423-428.

9. del Rio JC, Gutierrez A, Hernando M, Landin P, Romero J, Martinez AT: Determining the influence of eucalypt lignin composition in paper pulp yield using Py-GC/MS. J Anal Appl Pyrolysis 2005, 74:110-115.

10. Studer MH, DeMartini JD, Davis MF, Sykes RW, Davison B, Keller M, Tuskan GA Wyman CE: Lignin content in natural Populus variants affects sugar release. Proc Natl Acad Sci USA 2011, 108:6300-6305. S6300/6301-S6300/6306.

11. Li X, Ximenes E, Kim Y, Slininger M, Meilan R, Ladisch M, Chapple C: Lignin monomer composition affects Arabidopsis cell-wall degradability after liquid hot water pretreatment. Biotechnol Biofuels 2010, 3:27.

12. Lupoi JS, Singh S, Simmons BA, Henry RJ: Assessment of lignocellulosic biomass using analytical spectroscopy: an evolution to high-throughput techniques. Bioenerg Res 2014, 7:1-23.

13. Alves A, Simoes R, Stackpole DJ, Vaillancourt RE, Potts BM, Schwanninger M, Rodrigues J: Determination of the syringyl/guaiacyl ratio of Eucalyptus globulus wood lignin by near infrared-based partial least squares 
regression models using analytical pyrolysis as the reference method. I Near Infrared Spectrosc 2011, 19:343-348.

14. del Rio JC, Gutierrez A, Rodriguez IM, Ibarra D, Martinez AT: Composition of non-woody plant lignins and cinnamic acids by Py-GC/MS, Py/TMAH and FT-IR. J Anal Appl Pyrolysis 2007, 79:39-46.

15. Lupoi JS, Smith EA: Characterization of woody and herbaceous biomasses lignin composition with $1064 \mathrm{~nm}$ dispersive multichannel Raman spectroscopy. App/ Spectrosc 2012, 66:903-910.

16. Ona T, Sonoda T, Ito K, Shibata M, Katayama T, Kato T, Ootake Y: Nondestructive determination of lignin syringyl/guaiacyl monomeric composition in native wood by Fourier-transform Raman spectroscopy. J Wood Chem Technol 1998, 18:43-51.

17. Robinson AR, Mansfield SD: Rapid analysis of poplar lignin monomer composition by a streamlined thioacidolysis procedure and near-infrared reflectance-based prediction modeling. Plant J 2009, 58:706-714

18. Saariaho $A-M$, Jaaskelainen $A-S$, Nuopponen $M$, Vuorinen $T$ : Ultraviolet resonance Raman spectroscopy in lignin analysis: determination of characteristic vibrations of $p$-hydroxyphenyl, guaiacyl, and syringyl lignin structures. Appl Spectrosc 2003, 57:58-66.

19. Stackpole Desmond J, Vaillancourt Rene E, Alves A, Rodrigues J, Potts Brad M: Genetic variation in the chemical components of Eucalyptus globulus wood. G3 (Bethesda) 2011, 1:151-159.

20. Sun L, Varanasi P, Yang F, Loque D, Simmons BA, Singh S: Rapid determination of syringyl:guaiacyl ratios using FT-Raman spectroscopy. Biotechnol Bioeng 2012, 109:647-656.

21. Takayama M, Johjima T, Yamanaka T, Wariishi H, Tanaka H: Fouriertransform Raman assignment of guaiacyl and syringyl marker bands for lignin determination. Spectrochim Acta A Mol Biomol Spectrosc 1997, 53A:1621-1628.

22. Yamada T, Yeh T-F, Chang H-M, Li L, Kadla JF, Chiang VL: Rapid analysis of transgenic trees using transmittance near-infrared spectroscopy (NIR). Holzforschung 2006, 60:24-28.

23. Ona T, Sonoda T, Ohshima J, Yokota S, Yoshizawa N: A rapid quantitative method to assess Eucalyptus wood properties for kraft pulp production by FT-Raman spectroscopy. J Pulp Pap Sci 2003, 29:6-10.

24. Derkacheva OY: Estimation of aromatic structure contents in hardwood lignins from IR absorption spectra. J App/ Spectrosc 2013, 80:670-676.

25. Huang Y, Wang L, Chao Y, Nawawi DS, Akiyama T, Yokoyama T, Matsumoto $Y$ : Analysis of lignin aromatic structure in wood based on the IR spectrum. J Wood Chem Technol 2012, 32:294-303.

26. Sammons RJ, Harper DP, Labbe N, Bozell JJ, Elder T, Rials TG: Characterization of organosolv lignins using thermal and FT-IR spectroscopic analysis. BioResources 2013, 8:2752-2767.

27. McCreery RL: Raman Spectroscopy for Chemical Analysis. New York, USA: Wiley Interscience; 2000:448.

28. Meyer MW, Lupoi JS, Smith EA: $1064 \mathrm{~nm}$ dispersive multichannel Raman spectroscopy for the analysis of plant lignin. Anal Chim Acta 2011, 706:164-170.

29. Vitek P, Ali EMA, Edwards HGM, Jehlicka J, Cox R, Page K: Evaluation of portable Raman spectrometer with $1064 \mathrm{~nm}$ excitation for geological and forensic applications. Spectrochim Acta A Mol Biomol Spectrosc 2012, 86:320-327.

30. Smith W, Dent G: Modern Raman Spectroscopy. Chichester, UK: John Wiley \& Sons; 2005.

31. Workman JJ Jr: Interpretive spectroscopy for near infrared. App/ Spectrosc Rev 1996, 31:251-320.

32. Carroll A, Somerville C: Cellulosic biofuels. Annu Rev Plant Biol 2009 60:165-182.

33. Simmons BA: Bioenergy from plants and plant residues. In Plant Biotechnology and Agriculture: Prospects for the 21st Century. Edited by Altman AaH PM. Oxford: Academic; 2011:495-506.

34. Shepherd M, Bartle J, Lee DJ, Brawner J, Bush D, Turnbull P, MacDonel P, Brown TR, Simmons B, Henry R: Eucalypts as a biofuel feedstock. Biofuels 2011, 2:639-657.

35. Larsen KL, Barsberg S: Theoretical and Raman spectroscopic studies of phenolic lignin model monomers. J Phys Chem B 2010, 114:8009-8021.

36. Wiley JH, Atalla RH: Band assignments in the Raman spectra of celluloses. Carbohydr Res 1987, 160:113-129.

37. Agarwal UP, Atalla Rajai H: Vibrational Spectroscopy'. In Lignin and Lignans: Advances in Chemistry. Edited by Heitner C, Dimmel DR, Schmidt JA. Boca Raton,Florida: CRC Press; 2010:103-136.
38. Faix O: Classification of lignins from different botanical origins by FT-IR spectroscopy. Holzforschung 1991, 45:21-27.

39. Faix O: Fourier transform infrared spectroscopy [of lignin in solid state]'. In Methods in Lignin Chemistry. Edited by Lin SY, Dence CW. Berlin, Germany: Springer; 1992:83-109.

40. Kubo S, Kadla JF: Hydrogen bonding in lignin: a Fourier transform infrared model compound study. Biomacromolecules 2005, 6:2815-2821.

41. Bermello A, Del Valle M, Orea U, Carballo LR: Characterization by infrared spectrometry of lignins of three Eucalyptus species. Int J Polym Mater 2002, 51:557-566.

42. Barker B, Owen NL: Identifying softwoods and hardwoods by infrared spectroscopy. J Chem Educ 1999, 76:1706-1709.

43. Saariaho A-M, Argyropoulos DS, Jaeaeskelaeinen A-S, Vuorinen T: Development of the partial least squares models for the interpretation of the UV resonance Raman spectra of lignin model compounds. Vib Spectrosc 2005, 37:111-121.

44. Agarwal UP, MCSweeny JD, Ralph SA: FT-Raman investigation of milledwood lignins: softwood, hardwood, and chemically modified black spruce lignins. J Wood Chem Technol 2011, 31:324-344.

45. Schwanninger M, Rodrigues JC, Fackler K: A review of band assignments in near infrared spectra of wood and wood components. J Near Infrared Spectrosc 2011, 19:287-308

46. Workman J Jr, Weyer L (Eds): Practical Guide to Interpretive Near-Infrared Spectroscopy. Boca Raton, Florida: CRC Press; 2007.

47. Michell AJ, Schimleck LR: NIR spectroscopy of woods from Eucalyptus globulus. Appita J 1996, 49:23-26.

48. Shenk JS, Workman JJ Jr, Westerhaus MO: Application of NIR spectroscopy to agricultural products. Pract Spectrosc 2008, 35:347-386.

49. Alves AMM, Simoes RFS, Santos CA, Potts BM, Rodrigues J, Schwanninger M: Determination of Eucalyptus globulus wood extractives content by near infrared-based partial least squares regression models: comparison between extraction procedures. I Near Infrared Spectrosc 2012, 20:275-285.

50. Lee DJ: Achievements in forest tree genetic improvement in Australia and New Zealand 2: Development of Corymbia species and hybrids for plantations in eastern Australia. Aust For 2007, 70:11-16.

doi:10.1186/1754-6834-7-93

Cite this article as: Lupoi et al:: High-throughput prediction of eucalypt lignin syringyl/guaiacyl content using multivariate analysis: a comparison between mid-infrared, near-infrared, and Raman spectroscopies for model development. Biotechnology for Biofuels 2014 7:93.

\section{Submit your next manuscript to BioMed Central and take full advantage of:}

- Convenient online submission

- Thorough peer review

- No space constraints or color figure charges

- Immediate publication on acceptance

- Inclusion in PubMed, CAS, Scopus and Google Scholar

- Research which is freely available for redistribution 